\title{
Fast, economic and simultaneous identification of clinically relevant Gram-negative species with multiplex real-time PCR
}

\author{
Daniel Weiss*,1,2,3, Darius Gawlik², ${ }^{1, \text { Helmut Hotzel }}{ }^{4}$, Ines Engelmann ${ }^{1,2}$, Elke Mueller ${ }^{1,2}$, \\ Peter Slickers $^{1}$, Sascha D Braun ${ }^{1,2}$, Stefan Monecke ${ }^{1,2,5}$ \& Ralf Ehricht ${ }^{1,2,6}$ \\ ${ }^{1}$ Research \& Development, Abbott (Alere Technologies GmbH), Jena, Germany \\ ${ }^{2}$ Center for Applied Research, InfectoGnostics Research Campus, Jena, Germany \\ ${ }^{3}$ Institute for Infectious Diseases and Infection Control, University Medical Center of Jena, Jena, Germany \\ ${ }^{4}$ Institute of Bacterial Infections \& Zoonoses, Friedrich-Loeffler-Institute, Jena, Germany \\ ${ }^{5}$ Institute for Medical Microbiology \& Hygiene, Technical University of Dresden, Dresden, Germany \\ ${ }^{6}$ Department for Optical Molecular Diagnostics and Systems Technology, Leibniz-Institute of Photonic Technology, Jena, Germany \\ *Author for correspondence: Tel.: +0049 3641 3111254; daniel.weiss@alere.com
}

Aim: A newly designed multiplex real-time PCR (rt-PCR) was validated to detect four clinically relevant Gram-negative bacteria (Escherichia coli, Acinetobacter baumannii, Klebsiella pneumoniae and Pseudomonas aeruginosa). Materials \& methods: Serial dilutions of genomic DNA were used to determine the limit of detection. Colony PCR was performed with isolates of the four selected species and other species as negative controls. Isolates were characterized genotypically and phenotypically to evaluate the assay. Results: Specific signals of all target genes were detected with diluted templates comprising ten genomic equivalents. Using colony rt-PCR, all isolates of the target species were identified correctly. All negative control isolates were negative. Conclusion: The genes gad, basC, khe and ecfX can reliably identify these four species via multiplex colony rt-PCR.

First draft submitted: 29 June 2018; Accepted for publication: 22 November 2018; Published online: 12 December 2018

Keywords: colony PCR $\bullet$ Gram-negative bacteria $\bullet$ molecular species determination $\bullet$ multicolor detection $\bullet$ multiplex $\bullet$ real-time PCR • TaqMan

Gram-negative bacteria, such as Enterobacteriacae or nonfermenting bacteria, are responsible for a wide variety of different infections in humans, such as surgical/intraabdominal or urinary tract infections, ventilator-associated pneumonia and sepsis [1,2]. They often harbor different resistance and virulence mechanisms [1,3-6].

In recent years, the treatment of these infections became more complicated due to the emergence of Gram-negative bacteria that are resistant to a wide range of antibiotics. An overuse of antibiotics, especially of cephalosporins (third and fourth generation) in humans and livestock led to the emergence of Enterobacteriaceae and nonfermenters that produce extended spectrum $\beta$-lactamases [7]. Carbapenems are commonly and increasingly used as last line antibiotics for the therapy of infections with spectrum $\beta$-lactamases producers, or as initial treatment in urgent situations before the causative organism was identified and its susceptibility pattern was determined. The surging use of carbapenems consequently led to a selective pressure favoring different resistance mechanisms, for example, the production of various carbapenemases located mainly on mobile genetic elements. The prevalence of such multiresistant pathogens is continuously increasing worldwide [5,6]. Only a few antibiotics (e.g., colistin or fosfomycin) remain available for the treatment of carbapenem resistant Enterobacteriaceae and nonfermenters.

Recently published reports illustrated the prevalence of multiresistant Gram-negative bacteria on a global scale $[8,9]$. In 2017, the WHO published a list of bacteria in which resistance to the most common antibiotics is most critical [9]. The four most relevant species, in this regard, were considered as Escherichia coli, Klebsiella pneumoniae, Acinetobacter baumannii and Pseudomonas aeruginosa. Therefore, we focused on these four species.

Future Medicine 
An early identification and confirmation of suspicious pathogens beside its potential antimicrobial resistance is important and crucial for infection control, prevention, rapid confirmation of other diagnostic test procedures, treatment and epidemiological purposes [1].

Routine procedures in diagnostic laboratories for the identification of suspicious pathogens in cultures from patient samples are often time consuming, expensive, resource and labor intensive. Therefore, in a clinical environment, a lot of factors have to be considered such as restricted funds, time and staff. Isolates from patient samples are usually cultured on different growth media for further investigation. Different selective media are available that allow a preliminary identification (e.g., CHROMagar ${ }^{\mathrm{TM}}$ [10] or GCG-MUG agar [11]). A definite species identification is then achieved by phenotypic methods such as analysis of metabolic profiles (e.g., VITEK [8]), by MALDI-TOF [12], or by molecular means such as sequencing of $16 \mathrm{~S}$ rRNA [13] and/or rpoB [14]. In recent years, there was a constant increase in use of molecular methods for microbiological laboratories, such as PCR based (e.g., real-time PCR [15], etc.) or isothermal amplification methods (e.g., RPA [16-18], LAMP [16,18,19], NEAR [18,20] etc.) and also other recently developed methods that could lead to the development of new diagnostic tests to identify different Gram-negative species [21].

Our aim was to develop an accurate, specific and sensitive multiplex real-time PCR ( rt-PCR) to identify clinically most relevant Gram-negative bacteria (i.e., E. coli, K. pneumoniae, A. baumannii and P. aeruginosa) by using a fast and economic colony PCR. This technique allows obtaining a rapid result directly from a culture plate without further sample preparation and post-PCR analytical steps. It also should serve as a basis for a future development of rapid assays for the detection of target organisms directly from patient samples.

\section{Material \& methods \\ Strains \& isolates}

Characterized reference strains and clinical isolates used for this study were obtained from following sources: Alere in-house strain collection (Alere, Jena, Germany), German Collection of Microorganisms and Cell Cultures (DSMZ, Brunswick, Germany), University Medical Center of Dresden (UMC, Dresden, Germany) and the University of Fribourg (UF, Fribourg, Switzerland).

The limit of detection (LOD) was determined using the following reference strains; ID95726 (E. coli), ID204517 (P. aeruginosa), ID215765 (K. pneumoniae) and ID240770 (A. baumannii). More detailed information of all used reference strains are listed in the Supplementary Table 1.

Previously identified clinical isolates were used to determine the true positive rate (TPR; including: E. coli [n = 16], P. aeruginosa $[\mathrm{n}=15], K$. pneumoniae $[\mathrm{n}=18]$ and $A$. baumannii $[\mathrm{n}=16]$ ) and the TNR (including: $K$. oxytoca [n = 13], Citrobacter ssp. [n = 11], Enterobacter ssp. [n = 10], Chryseobacterium ssp. [n= 4], Serratia ssp. [n = 4], Stenotrophomas ssp. [n = 4], Elizabethkingia meningoseptica [n = 2], Acinetobacter baylyi, Acinetobacter junii and Acinetobacter Iwoffii, [each n = 1], Aeromonas spec. [n = 1], Proteus spec. [n =1], P. oleovorans $[\mathrm{n}=1]$ and Pseudomonas stutzeri [each $\mathrm{n}=1]$ ). For more details, see also Supplementary Table 1 (TPR) and Supplementary Table 2 (TNR).

All reference strains and isolates were cultured on tryptone yeast agar at $37^{\circ} \mathrm{C}$ for $16 \mathrm{~h}$.

\section{Reference methods for identification}

All isolates were characterized phenotypically by using the Bruker Daltonics MALDI Biotyper (Bruker Daltonik GmbH, Bremen, Germany) and the VITEK-2 system (bioMerieux, Nürtingen, Germany). The Bruker MALDITOF system was the primary method for species identification. Briefly, a tiny colony of each isolate was picked and suspended in $300 \mu \mathrm{l}$ of water. Subsequently, $900 \mu \mathrm{l}$ of absolute alcohol were added, mixed and the suspensions were stored at $-20^{\circ} \mathrm{C}$ for further usage. Such prepared suspensions were tested at least two-times to evaluate the results. All data were obtained using the Compass 1.4 Software and BioTyper database 3.0 for species identification (Bruker Daltonik GmbH, Bremen, Germany).

If divergent results were obtained during different experiments with the same isolate solutions, the VITEK-2 system was used as second method for species identification (VITEK card: VITEK ${ }^{\circledR} 2$ GN ID). VITEK analyses were conducted according to the manufacturer's instructions. The data were analyzed automatically using to the VITEK-2 software [22].

Genotypic characterizations were carried out using DNA microarrays. Therefore labeling, hybridization and analysis were executed with the Abbott CarbDetect AS-2 Kit (Abbott, Jena, Germany) according to the manufacturer's instructions. For experimental details refer to Braun et al. 2014 and 2018 [3,23]. 


\begin{tabular}{|c|c|c|c|c|}
\hline Name & Sequence $\left(5^{\prime}-3^{\prime}\right)$ & $\operatorname{Tm}\left({ }^{\circ} \mathrm{C}\right)$ & Amplicon (bp) & Gene accession number \\
\hline khe-FW & TGGGGATCCACCACGA & 53.0 & \multirow[t]{3}{*}{126} & \multirow[t]{3}{*}{ AF293352.1 } \\
\hline khe -RV & AGAGATAGCCGTTTATCCACAC & 55.0 & & \\
\hline khe -TMP & 6Fam-GAGGAAGAGTTCATCTACGTGCTGGAGG-BHQ1 & 63.4 & & \\
\hline ecfX-FW & ATGAGCGCTTCCGTGGTTC & 58.1 & \multirow[t]{3}{*}{67} & \multirow[t]{3}{*}{ DQ996558.1 } \\
\hline ecfX-RV & AGGAAGCGCAGCAACTCG & 58.4 & & \\
\hline ecfX-TMP & HEX-TCTCGCATGCCTATCAGGCGTTCCAT-BHQ1 & 65.3 & & \\
\hline basC-FW & CTTGGTTACTATGGCCAATCC & 53.0 & \multirow[t]{3}{*}{153} & \multirow[t]{3}{*}{ CP000521.1 } \\
\hline basC-RV & GGTAATTGTTTTGAAGCCCA & 52.0 & & \\
\hline basC-TMP & ROX-CCACGCCGTGAATATGACCATTATTG-BHQ2 & 60.0 & & \\
\hline gad-FW & GGATATCGTCTGGGACTTCCG & 57.0 & \multirow[t]{3}{*}{77} & \multirow[t]{3}{*}{ AE014075.1 } \\
\hline gad-RV & GCGGAGCCAGACCGAATTT & 58.0 & & \\
\hline gad-TMP & Cy5-GTGAAATCGATCAGTGCTTCAGGCCA-BHQ3 & 62.8 & & \\
\hline
\end{tabular}

\section{Genomic DNA preparation}

For DNA preparation, the Qiagen DNeasy Blood \& Tissue Kit (Qiagen GmbH, Hilden, Germany) was used according to manufacturer's instructions. The DNA concentrations were spectrophotometrically determined by Nanodrop ND-1000 (Thermo Fisher Scientific, Dreieich, Germany). Genomic equivalents (GE) were calculated using the DNA concentration $[\mathrm{ng} / \mu \mathrm{l}]$, the mean genome size of species to be detected $(A$. baumannii: 4.0 mega base pairs [Mbp]; P. aeruginosa: $6.6 \mathrm{Mbp}$; E. coli: $5.2 \mathrm{Mbp}$ K pneumoniae: $5.5 \mathrm{Mbp}$ ), the mean molecular weight of the deoxynucleoside monophosphates $\left(32,695 \mathrm{~g} \mathrm{~mol}^{-1}\right)$ and the Avogadro constant $\left(6.023 \times 10^{23} \mathrm{~mol}^{-1}\right)$.

Genomic equivalents [genome copies $\times \mu \mathrm{l}^{-1}$ ] were calculated by multiplying the determined DNA concentration with the Avogadro constant and divide the result by the molecular weight of the genome, which is calculated by the number of bases multiplied with the mean molecular weight of the deoxynucleoside monophosphates. Subsequently, dilution series of all reference strains were prepared to cover a range of $10^{7}$ to $10 \mathrm{GE}$.

\section{Design of primers \& probes \& rt-PCR conditions}

The following genes were selected: $\operatorname{gad}(E$. coli), ecfX (P. aeruginosa), bas $C$ (A. baumannii) and khe (K. pneumoniae). Primer and probes were designed using consensus sequence areas of the target genes and their alleles to ensure the accuracy of the assay. Abbott primer design software package (Abbott, Jena, Germany) was used to design the primer and TaqMan probes. Special attention was placed on highly conserved regions of each species marker gene to cover all known alleles, which were published at time of the assay design (February 2017). Briefly, all GenBank entries for any given target were retrieved and one proofed and published entry was selected as reference sequence (Table 1). The resulting BLAST hits were reannotated and archived into a local database. Sequences were classified into paralogs and allelic variants based on similarity. For this, all matching regions from the alignments were used for the design of probes and primers. The sequences were selected which specifically have a similar GC content, length and melting temperature. Afterward, all designed sequences were reblasted against all available target sequences to rule out false negative or cross-reactive binding events. Subsequently, TaqMan probes were designed with different fluorophores for each target gene, in other words, Cy5 (red), ROX (orange), HEX (yellow) and 6FAM (green), which were covalently attached to the $5^{\prime}$-end of the sequence. Each target was evaluated in single- and multiplex reactions. The primers and TaqMan probes were synthetized by metabion international AG (Planegg/Steinkirchen, Germany). Detailed information about the used primers and probes (sequences, melting temperature, amplicon size) as well as the target genes (accession numbers) are provided in Table 1.

The assays were carried out using the RNA UltraSense ${ }^{T M}$ One-Step Quantitative RT-PCR System Kit (ThermoFischer, Dreieich, Germany), where $25 \mu$ reaction volumes were used containing $200 \mathrm{nM}$ of each primer and TaqMan probe. At last, $2 \mu \mathrm{l}$ of prepared DNA were added to the reaction mix. The rt-PCRs were performed using a Corbett Rotor-Gene 6000 Cycler (Qiagen, Hilden, Germany). The PCR program consisted of the following steps: 2 min of initial denaturation at $94^{\circ} \mathrm{C}$ followed by 40 cycles of denaturation at $94^{\circ} \mathrm{C}$ for $10 \mathrm{~s}$, annealing at $55^{\circ} \mathrm{C}$ for $30 \mathrm{~s}$ and extension at $72^{\circ} \mathrm{C}$ for $30 \mathrm{~s}$. All data were collected during the annealing phase and subsequently analyzed using the Rotor-Gene 6000 software 2.3.1 (Qiagen GmbH, Hilden, Germany). 
For colony PCR, the isolates were cultured on tryptone yeast agar for $16 \mathrm{~h}$ at $37^{\circ} \mathrm{C}$. A single colony of each isolate was picked and suspended in $10 \mu \mathrm{l}$ of molecular grade water. After heating at $95^{\circ} \mathrm{C}$ for $10 \mathrm{~min}$, the suspension was added directly to the $15 \mu \mathrm{l}$ of the PCR reaction mix. The final concentrations of TaqMan probes and primer were identical as described above. Please note that for colony rt-PCR, the PCR program consisted of 35 cycles only.

\section{Limit of detection \& PCR efficiency}

The LOD was determined in single- and multiplex reactions using three replicates of a dilution series covering a range of $10^{7}$ to $10 \mathrm{GE}$. For the standard curves the mean threshold cycle $\left(\mathrm{C}_{\mathrm{T}}\right)$ values of the three replicates of each dilution were plotted against the log concentration of the gDNA template.

The rt-PCR efficiencies (E) were calculated from the slopes $(\mathrm{S})$ of the linear regressions of $\mathrm{C}_{\mathrm{T}}$ standard curves (Figure 1), as described previously [24].

$$
E=10^{\left(-\frac{1}{S}\right)}-1
$$

The linearity of the $\mathrm{C}_{\mathrm{T}}$ standard curves was verified using the coefficients of determination $\left(\mathrm{R}^{2}\right)$ which were calculated from the linear regression curves using the $\log _{10}$ copy numbers $(\mathrm{x})$, the mean $\mathrm{C}_{\mathrm{T}}$ values of the three replicates (y) and the quantity of measured values (n).

$$
R^{2}=\left(\frac{\sum x y-\frac{1}{n}\left(\sum x\right)\left(\sum y\right)}{\sqrt{\left[\sum x^{2}-\frac{1}{n}\left(\sum x\right)^{2}\right]\left[\sum y^{2}-\frac{1}{n}\left(\sum y\right)^{2}\right]}}\right)^{2}
$$

True positive \& negative rates

True positive and negative were calculated to analyze the performance of this assay in comparison with the alternative methods used in this paper.

The TPR measures the proportion of the true positive tested isolates (TP) that are correctly identified in this assay to the overall positive isolates $(\mathrm{P})$ which include the true positive and false negative isolates $(\mathrm{FN})$.

$$
\text { true positive rate }(T P R)=\frac{T P}{P}=\frac{T P}{T P+F N}
$$

TNR measures the proportion of the true negative isolates (TN) that are correctly identified in this assay to the overall negative isolates $(\mathrm{N})$. The negative isolates include the true negative isolates and the false positive isolates (FP) which were detected in this assay.

$$
\text { true negative rate }(T N R)=\frac{T N}{N}=\frac{T N}{T N+F P}
$$

\section{Results}

The LOD for all target genes was detected in single- and multiplex reactions. In both cases, a LOD of 10 GE for $k$ he, bas $C$ and gad was determined (Figure 1). The species marker gene ecf $X$ which could only be detected to gene copies comprising as $10^{2} \mathrm{GE}$ of template DNA (Figure 1).

The PCR efficiency of the target genes were in singleplex $90.4 \%(\mathrm{khe}), 101.6 \%(\mathrm{bas}), 96.7 \%(\mathrm{gad})$ and 86.8\% (ecfX) and in multiplex 83.1\% (khe), 95.1\% (bas C), 92.3\% ( gad) and 88.5\% (ecfX). The coefficients of determination $\left(\mathrm{R}^{2}\right)$ were in all cases $\mathrm{R}^{2}>0.99$, which demonstrated a high linearity of the $\mathrm{C}_{\mathrm{T}}$ standard curves (Figure 1).

All 65 isolates which carried one of the selected species markers were correctly identified (Table 2; Supplementary Table 3). Furthermore, no false negative results were observed when using the multiplex approach. It was also confirmed that the 55 examined isolates of the other species did not harbor the selected target species markers 
(A) Singleplex real-time PCR
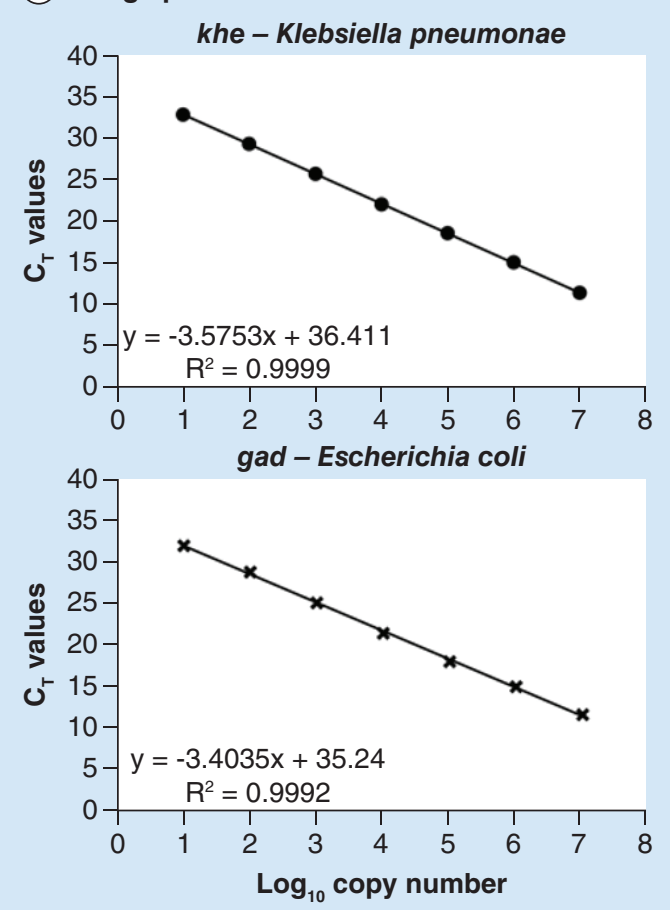

(B) Multiplex real-time PCR
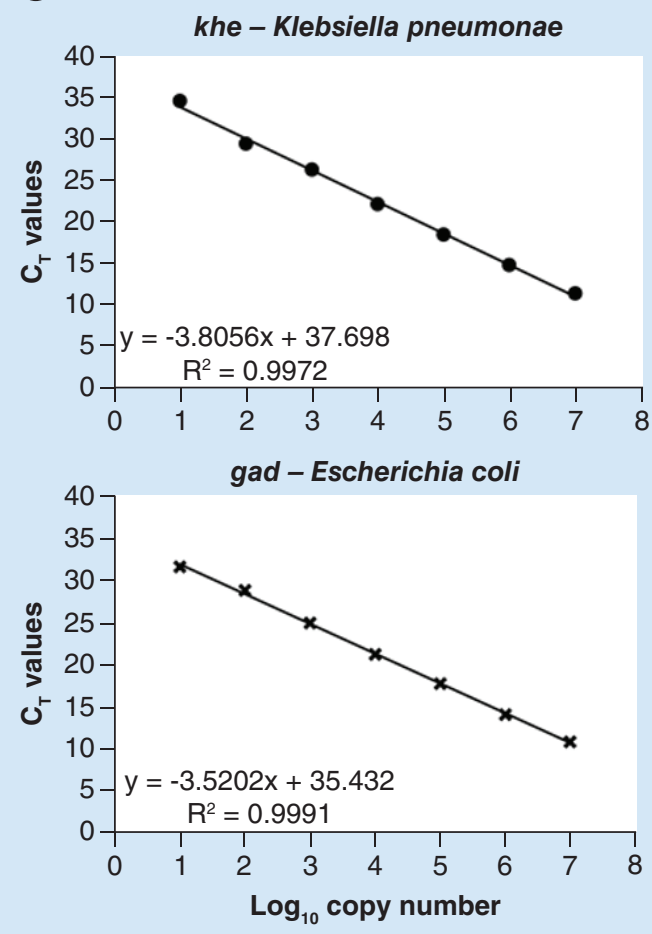
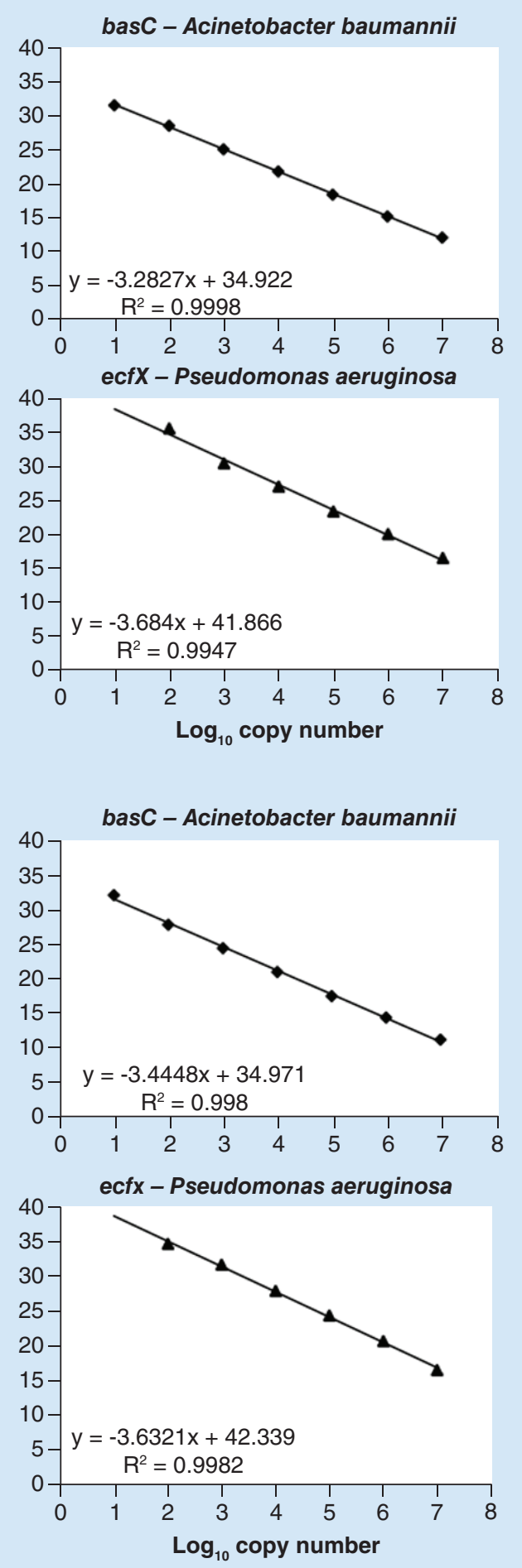

Figure 1. Standard curves of TaqMan-probe for singleplex and multiplex reactions. Standard curves of TaqMan-probe based multiplex real time-PCR using DNA copies in a range from $10^{7}$ to $10 \mathrm{GE}(\mathrm{n}=3)$ of reference strains (Escherichia coli [strain ID: 95726]; Klebsiella pneumoniae [strain ID: 215765]; Acinetobacter baumannii [strain ID: 240770] and Pseudomonas aeruginosa [strain ID: 204517]) in (A) singleplex and (B) multiplex reactions were generated to compare the initial number of DNA copies with their threshold cycle of detection. 


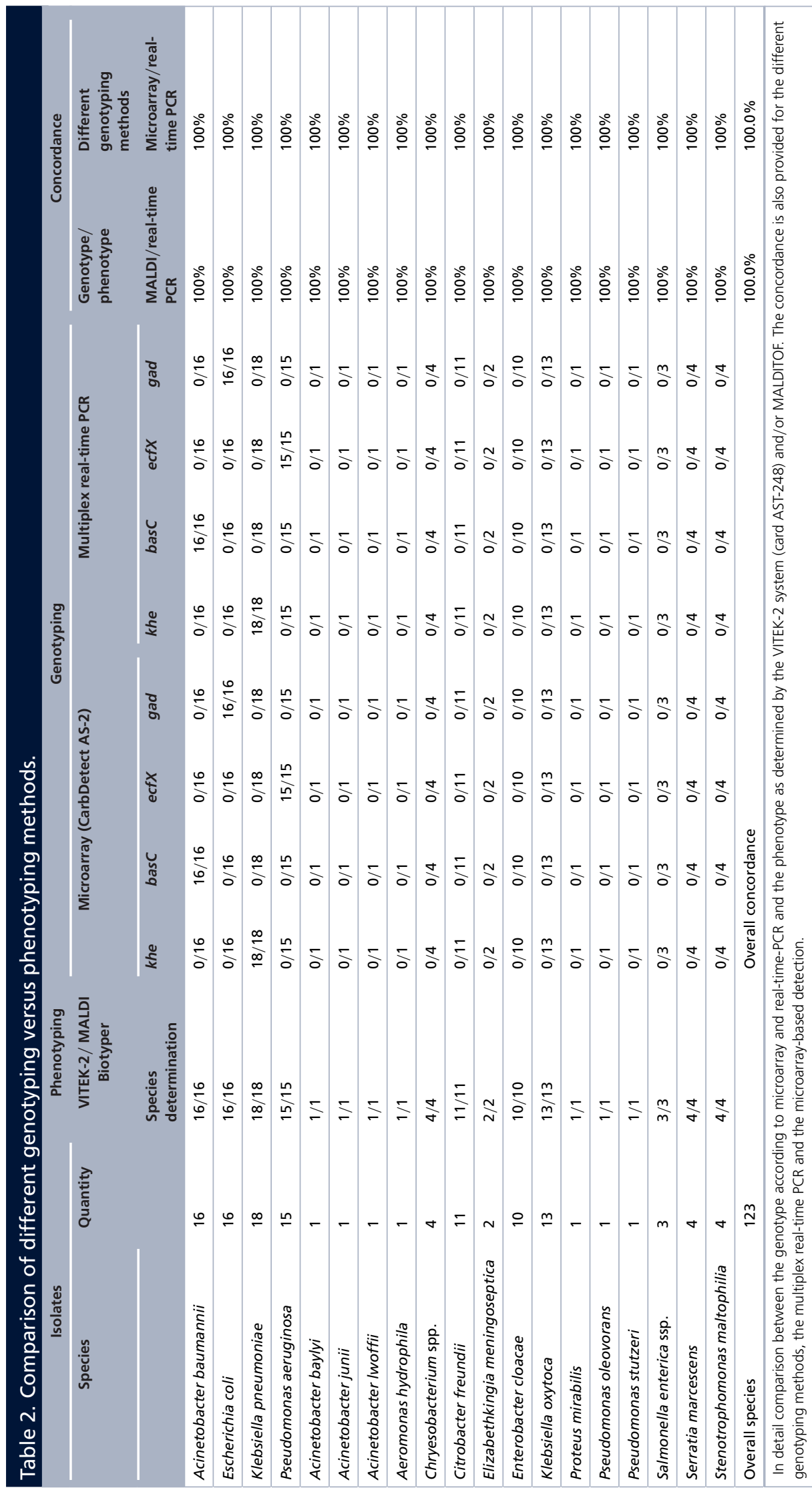


(Table 2; Supplementary Table 3). Therefore, the true positive rate and true negative rate of this assay were both $100 \%$.

The concordance of this multiplex rt-PCR assay in comparison to the microarray and VITEK-2/MALDI systems was ,therefore, in both cases $100 \%$.

\section{Discussion}

In recent years, several rt-PCRs were established to detect the most clinical important Gram-negative bacteria, E. coli, A. baumannii, K. pneumoniae and P. aeruginosa [9]. In most of these approaches, SYBR-green staining was used $[25,26]$ and the different targets were afterward analyzed using melting curve assays. The SYBR green binds unspecifically to single-stranded DNA, double-stranded DNA and RNA, and the resulting fluorescence can only be detected in the green fluorescence channel. Therefore, it does not allow a precise distinction between different target genes or possible secondary products in a multiplex real-time assay. For that reason, the SYBR-green staining was not the method of choice for a multiplex-based assay as described in this paper. A TaqMan probes based rt-PCR was more suitable for multiplex applications since target genes can be detected at different fluorescence channels, which excludes an overlap of signals of other targets as well as of possible secondary products. Since melting curves analysis with different melting temperatures of the amplicons were not necessary for a precise differentiation of the target genes in our TaqMan probe real-time assay, amplicon sizes were kept relatively short (67-153 bp). For a possible point of care application in the future short amplicon sizes are advantageous, as native patient samples could contain fragmented DNA.

The LOD of all target genes were detected in single- and multiplex reactions using a DNA dilution series of reference strains diluted down to ten genomic equivalents. An exception was ecf $X$ which could only be detected in dilutions containing $10^{2} \mathrm{GE}$. Although the PCR efficiency of the targets khe, bas $C$ and gad decreased in the multiplex reaction, which was to be expected as more different primers were present in the reaction, no significant difference was observed regarding the LOD. In contrast to the other three targets, ecf $X$ demonstrated a higher PCR efficiency in multiplex reactions compared with the singleplex reactions. The target bas $C$ showed a PCR efficiency of slightly over $100 \%$ in singleplex reactions. It was reported in several rt-PCR assays that PCR efficiencies up to $110 \%$ were acceptable [27-29]. Since the calculation is based on the slopes of the linear regression of the standard curves, the slopes can be influenced by inhibitory effects in the PCR reaction, PCR conditions or divergences in the DNA copies of the serial dilutions [24]. That occurs especially in the higher diluted DNA solutions, where even small differences in gene copies lead to significant variations in the resulting $\mathrm{C}_{\mathrm{T}}$ values. Handling conditions as calibration of the pipets or the pipetting technique might also have some significance [30]. Even mathematical factors (e.g., measuring errors, reading errors, round ups or the assignment of the threshold line) might have a significant influence to the standard curve and regression slope. Since the deviation was just $1.6 \%$ the results were tolerated. The coefficients of determination of the regression slopes demonstrated a high linearity of the standard curves in single- and multiplex reactions of each target gene.

The results of the colony multiplex PCR revealed that gad, khe, bas $C$ and ecf $X$ were reliable genes for species identification in clinical isolates being specific for each of the four target species. Other, related species belonging to the same genera were tested negative. True positive rate and true negative rate are important to analyze the performance of assays in comparison with other methods. The TPR and TNR were both 100\% which illustrated the accuracy of this assay. Using colony PCRs by picking colonies directly from agar plate, it was demonstrated that prior DNA preparations were not necessary saving costs and efforts.

\section{Conclusion}

The results of this study revealed a high TPR, TNR and LOD of the assay for all target genes. The targets can be detected rapidly and specifically via colony rt-PCR. The LOD was low enough to consider future application to native patient samples. Furthermore, this method is time and cost effective since it can be performed within less than $2 \mathrm{~h}$ and up to four target genes can be detected in one reaction simultaneously. Colonies of isolates can be directly used from agar plates; therefore, time-consuming steps as the preparation of DNA were not necessary. This simple method demonstrated that it can be employed directly in most laboratories, where the necessary PCR equipment is available. This multiplex rt-PCR could be used as a rapid test in order to confirm or identify the target species, for example, isolated from selective media or from positive blood cultures. In combination with the carbapenemase multiplex rt-PCR published by Weiss et al. 2017 [1], this assay could be a tool analyzing conspicuous isolates $[1,6]$. 
Both assays could also be used to develop a lab-on-a-chip point of care test for the screening of species and carbapenemases genes in native patient samples. One technology to achieve this goal could be the competitive reporter monitored amplification, which combines microarray technology with real-time detection [31].

\section{Summary points}

- A novel multiplex real time-PCR (rt-PCR) was designed, developed and validated to detect the four clinically relevant Gram-negative bacteria targeting the species specific genes: gad (Escherichia coli), khe (Klebsiella pneumoniae), basC (Acinetobacter baumannii) and ecfX (Pseudomonas aeruginosa) without DNA preparation.

- Primer and TaqMan probes were designed targeting conserved sequence regions for each target and covering all currently known alleles to ensure high accuracy of the assay.

- The limit of detection was evaluated using dilution series of DNA of reference strains with a predetermined number of genome copies.

- The true positive and negative rates of the assay were analyzed using colony PCR of 65 isolates of the four species considered, as well as of 55 negative controls belonging to other species.

- Species identifications by rt-PCR were compared with identifications obtained in parallel using microarray based assays (CarbDetect AS-2, Alere), VITEK-2 (BioMerieux) and MALDI-TOF (Bruker) systems.

- Specific signals for all selected targets were detected with genomic DNA diluted down to ten genomic equivalents. Using a dilution series of DNA of the four species combined, the multiplex reaction targeting all four markers was shown to have the same limit of detection as the corresponding individual reactions.

- Using colony rt-PCR, all isolates of the target species were identified correctly and all isolates of other species and negative controls gave negative results. The concordance of the rt-PCR assay to the other methods was $100 \%$.

- The genes gad, basC, khe and ecfX can reliably be identified via colony rt-PCR in a single reaction.

- Colonies can be used directly from agar plates saving costs and time for DNA preparation and the assay does not require post-PCR sample handling.

- This multiplex rt-PCR could be used as rapid test to confirm or identify the target species, for example, isolated from selective media or blood cultures.

Supplementary data

To view the supplementary data that accompany this paper please visit the journal website at: https://www.futuremedicine.com/d oi/suppl/10.2217/fmb-2018-0197

\section{Acknowledgements}

The authors gratefully acknowledge the InfectoGnostics Research Campus Jena for support. We acknowledge Peggy Methner at the Friedrich-Loeffler-Institute, Jena, Germany, for help with MALDI-TOF analyses.

Financial disclosure \& competing interests

All mentioned authors are employees of Abbott (Alere Technologies $\mathrm{GmbH}$ ), but this had no influence on study design and execution. The authors have no other relevant affiliations or financial involvement with any organization or entity with a financial interest in or financial conflict with the subject matter or materials discussed in the manuscript apart from those disclosed.

No writing assistance was utilized in the production of this manuscript.

Ethics conduct of research

Ethic committees consent was not requested since isolates were not purposefully obtained for this study but retrospectively selected from a strain collection. No patient data were stored or utilized for this study.

\section{Open access}

This work is licensed under the Attribution-NonCommercial-NoDerivatives 4.0 Unported License. To view a copy of this license, visit http://creativecommons.org/licenses/by-nc-nd/4.0/ 


\section{References}

Papers of special note have been highlighted as: $\bullet$ of interest; $\bullet \bullet$ of considerable interest

1. Weiss D, Engelmann I, Braun SD, Monecke S, Ehricht R. A multiplex real-time PCR for the direct, fast, economic and simultaneous detection of the carbapenemase genes blaKPC, blaNDM, blaVIM and blaOXA-48. J. Microbiol. Methods 142, 20-26 (2017).

-• A short communication paper which represents the first part of this work. Therein, the same method was used as described in this publication, but carbapenemases genes were chosen as targets.

2. Gaynes R, Edwards JR. Overview of nosocomial infections caused by gram-negative bacilli. Clin. Infect. Dis. 41(6), $848-854$ (2005).

3. Braun SD, Monecke S, Thurmer A et al. Rapid identification of carbapenemase genes in gram-negative bacteria with an oligonucleotide microarray-based assay. PLoS ONE 9(7), 1-11 (2014).

-. Describes in more detail the complete method of the microarray-based assay (CarbDetect AS-2 Kit) used in this study.

4. Dallenne C, Da Costa A, Decre D, Favier C, Arlet G. Development of a set of multiplex PCR assays for the detection of genes encoding important beta-lactamases in Enterobacteriaceae. J. Antimicrob. Chemother. 65(3), 490-495 (2010).

5. Poirel L, Walsh TR, Cuvillier V, Nordmann P. Multiplex PCR for detection of acquired carbapenemase genes. Diagn. Microbiol. Infect. Dis. 70(1), 119-123 (2011).

6. Veeraraghavan B, Shankar C, Karunasree S, Kumari S, Ravi R, Ralph R. Carbapenem resistant Klebsiella pneumoniae isolated from bloodstream infection: Indian experience. Pathog. Glob. Health 111(5), 240-246 (2017).

7. Landers TF, Cohen B, Wittum TE, Larson EL. A review of antibiotic use in food animals: perspective, policy, and potential. Public Health Rep. 127(1), 4-22 (2012).

8. Osei Sekyere J, Govinden U, Essack SY. Review of established and innovative detection methods for carbapenemase-producing Gram-negative bacteria. J. Appl. Microbiol. 119(5), 1219-1233 (2015).

- Points out advantages and disadvantages of standard methods and newly available methods regarding the detection of carbapenemase-producing Gram-negative bacteria.

9. Tacconelli E, Magrini N. Global priority list of antibiotic-resistant bacteria to guide research, discovery, and development of new antibiotics. WHO (2017). www.who.int/medicines/publications/WHO-PPL-Short_Summary_25Feb-ET_NM_WHO.pdf?ua=1

-• WHO published a list of as 'critical' referred Gram-negative bacteria which are resistant to most commonly used antibiotics. This article led to the choice of the target species used for the development of the herein described assay.

10. Hornsey M, Phee L, Woodford N et al. Evaluation of three selective chromogenic media, CHROMagar ESBL, CHROMagar CTX-M and CHROMagar KPC, for the detection of Klebsiella pneumoniae producing OXA-48 carbapenemase. J. Clin. Pathol. 66(4), 348-350 (2013).

11. Ziesche K, Reissbrodt R, Rische H. [A bile-chrysoidine-glycerol culture medium and its use in the diagnosis of gram-negative aerobic bacteria, especially Enterobacteriaceae]. Z. Gesamte Hyg. 31(9), 516-518 (1985).

12. Singhal N, Kumar M, Kanaujia PK, Virdi JS. MALDI-TOF mass spectrometry: an emerging technology for microbial identification and diagnosis. Front. Microbiol. 6, 791 (2015).

-• Describes species identification using MALDI-TOF mass spectrometry, which was used as phenotyping method in this study.

13. Shin HB, Yoon J, Lee Y, Kim MS, Lee K. Comparison of MALDI-TOF MS, housekeeping gene sequencing, and 16S rRNA gene sequencing for identification of Aeromonas clinical isolates. Yonsei Med. J. 56(2), 550-555 (2015).

14. Mollet C, Drancourt M, Raoult D. rpoB sequence analysis as a novel basis for bacterial identification. Mol. Microbiol. 26(5), 1005-1011 (1997).

15. Espy MJ, Uhl JR, Sloan LM et al. Real-time PCR in clinical microbiology: applications for routine laboratory testing. Clin. Microbiol. Rev. 19(1), 165-256 (2006).

16. Maffert P, Reverchon S, Nasser W, Rozand C, Abaibou H. New nucleic acid testing devices to diagnose infectious diseases in resource-limited settings. Eur. J. Clin. Microbiol. Infect. Dis. 36(10), 1717-1731 (2017).

17. Piepenburg O, Williams CH, Stemple DL, Armes NA. DNA detection using recombination proteins. PLoS Biol. 4(7), e204 (2006).

18. Gill P, Ghaemi A. Nucleic acid isothermal amplification technologies: a review. Nucleosides Nucleotides Nucleic Acids 27(3), 224-243 (2008).

19. Salisu IB, Shahid AA, Yaqoob A et al. Molecular approaches for high throughput detection and quantification of genetically modified crops: a review. Front. Plant Sci. 8, 1670 (2017).

20. Persano S, Guevara ML, Wolfram J et al. Label-free isothermal amplification assay for specific and highly sensitive colorimetric miRNA detection. ACS Omega 1(3), 448-455 (2016).

21. Ipe DS, Sundac L, Benjamin WH Jr, Moore KH, Ulett GC. Asymptomatic bacteriuria: prevalence rates of causal microorganisms, etiology of infection in different patient populations, and recent advances in molecular detection. FEMS Microbiol. Lett. 346(1), 1-10 (2013).

22. Pincus DH. Microbial identification using the bioMérieux Vitek ${ }^{\circledR} 2$ system. In: Encyclopedia of Rapid Microbiological Methods. Miller MJ (Ed.). Parenteral Drug Association, MD, USA (2006). https://store.pda.org/tableofcontents/ermm_v2_ch01.pdf 
-. The complete method of the VITEK-2 system is described in this article, which was used as second phenotyping method in this study.

23. Braun SD, Jamil B, Syed MA et al. Prevalence of carbapenemase-producing organisms at the Kidney Center of Rawalpindi (Pakistan) and evaluation of an advanced molecular microarray-based carbapenemase assay. Future Microbiol. 13, 1225-1246 (2018).

-. The first part of this article describes the evaluation of the new updated CarbDetect AS-2 microarray, which was used for the genotyping in this publication.

24. Svec D, Tichopad A, Novosadova V, Pfaffl MW, Kubista M. How good is a PCR efficiency estimate: recommendations for precise and robust qPCR efficiency assessments. Biomol. Detect. Quantif. 3, 9-16 (2015).

25. Anbazhagan D, Mui WS, Mansor M, Yan GO, Yusof MY, Sekaran SD. Development of conventional and real-time multiplex PCR assays for the detection of nosocomial pathogens. Braz. J. Microbiol. 42(2), 448-458 (2011).

26. Clifford RJ, Milillo M, Prestwood J et al. Detection of bacterial $16 \mathrm{~S}$ rRNA and identification of four clinically important bacteria by real-time PCR. PLoS ONE 7(11), 1-6 (2012).

27. D'haene B, Vandesompele J, Hellemans J. Accurate and objective copy number profiling using real-time quantitative PCR. Methods 50(4), 262-270 (2010).

28. Gadsby NJ, McHugh MP, Russell CD et al. Development of two real-time multiplex PCR assays for the detection and quantification of eight key bacterial pathogens in lower respiratory tract infections. Clin. Microbiol. Infect. 21(8), 788 e781-788 e713 (2015).

29. Garson JA, Huggett JF, Bustin SA et al. Unreliable real-time PCR analysis of human endogenous retrovirus-W (HERV-W) RNA expression and DNA copy number in multiple sclerosis. AIDS Res. Hum. Retroviruses 25(3), 377-378; author reply 379-381 (2009).

30. Taylor S, Wakem M, Dijkman G, Alsarraj M, Nguyen M. A practical approach to RT-qPCR-Publishing data that conform to the MIQE guidelines. Methods 50(4), 1-5 (2010)

31. Ullrich T, Ermantraut E, Schulz T, Steinmetzer K. Competitive reporter monitored amplification (CMA) - quantification of molecular targets by real time monitoring of competitive reporter hybridization. PLoS ONE 7(4), e35438 (2012).

- A complete description of the reporter monitored amplification as an innovative technology. It may allow a possible future development for a lab-on-the-chip point of care test using the herein described assay. 